\title{
The all-diode-pumped laser system POLARIS - an experimentalist's tool generating ultra-high contrast pulses with high energy
}

Marco Hornung ${ }^{1,2}$, Hartmut Liebetrau ${ }^{2}$, Andreas Seidel ${ }^{2}$, Sebastian Keppler ${ }^{2}$, Alexander Kessler ${ }^{1}$, Jörg Körner ${ }^{2}$, Marco Hellwing ${ }^{2}$, Frank Schorcht ${ }^{1}$, Diethard Klöpfel ${ }^{2}$, Ajay K. Arunachalam ${ }^{1}$, Georg A. Becker $^{2}$, Alexander Sävert ${ }^{1,2}$, Jens Polz ${ }^{2}$, Joachim Hein ${ }^{1,2}$, and Malte C. Kaluza ${ }^{1,2}$

${ }^{1}$ Helmholtz-Institute Jena, Germany

${ }^{2}$ Institute of Optics and Quantum Electronics, Jena, Germany

(Received 28 February 2014; revised 25 April 2014; accepted 6 June 2014)

\begin{abstract}
The development, the underlying technology and the current status of the fully diode-pumped solid-state laser system POLARIS is reviewed. Currently, the POLARIS system delivers $4 \mathrm{~J}$ energy, 144 fs long laser pulses with an ultra-high temporal contrast of $5 \times 10^{12}$ for the ASE, which is achieved using a so-called double chirped-pulse amplification scheme and cross-polarized wave generation pulse cleaning. By tightly focusing, the peak intensity exceeds $3.5 \times 10^{20} \mathrm{~W} \mathrm{~cm}^{-2}$. These parameters predestine POLARIS as a scientific tool well suited for sophisticated experiments, as exemplified by presenting measurements of accelerated proton energies. Recently, an additional amplifier has been added to the laser chain. In the ramp-up phase, pulses from this amplifier are not yet compressed and have not yet reached the anticipated energy. Nevertheless, an output energy of $16.6 \mathrm{~J}$ has been achieved so far.
\end{abstract}

Keywords: design; high power laser; laser amplifiers; laser plasmas interaction; laser systems; modelling; optimization; ultra-intense; ultrashort pulse laser interaction with matter

\section{Introduction}

Chirped-pulse amplification ( $\left.\mathrm{CPA}^{[1]}\right)$ laser systems with output powers of several terawatts or even petawatts, which can be focused to intensities in excess of $10^{22} \mathrm{~W} \mathrm{~cm}^{-2}$ are widely used to study laser-matter interactions. During the past three decades this field of science has been growing rapidly and it has been shown that the laser performance in terms of pulse duration, pulse energy, and temporal intensity contrast strongly affects the experimental results. Experiments of particular interest are electron ${ }^{[2,3]}$ or ion acceleration $^{[4]}$, the laser-based generation of X-rays ${ }^{[5]}$, highenergy physics ${ }^{[6]}$ or laser-based proton radiography ${ }^{[4,7]}$. The community interested in these high-intensity phenomena is spread worldwide, operating several dozens of high-intensity lasers, the majority of which are based on direct (e.g., for Nd:Glass-systems) or indirect (e.g., for Ti:Sapphire-systems, which are pumped by flashlamppumped frequency-doubled Nd:YAG Lasers) pumping of the active material by flash lamps.

Correspondence to: Dr. Marco Hornung, Helmholtz-Institute Jena, Fröbelstieg 3, 07743 Jena, Germany. Email: Marco.Hornung@uni-jena.de
However, for more than one decade strong efforts have been made to establish diode-pumped solid-sate laser (DPSSL) technology for generating high-energy femtosecond or picosecond laser pulses ${ }^{[8]}$. The most commonly used $\mathrm{Yb}^{3+}$-doped amplification media have already been used for the amplification of ns-laser pulses to energies in excess of $10 \mathrm{~J}$, as recently shown in the projects LUCIA (Yb:YAG, $14 \mathrm{~J}^{[9]}$ ), DIPOLE (Yb:YAG, $10 \mathrm{~J}^{[10]}$ ), and MERCURY $\left(\mathrm{Yb}: S-F A P, 55 \mathrm{~J}^{[11]}\right)$. Furthermore, a number of projects have started to investigate and to develop high-energy class DPSSLs (HECDPSSL) all over the world ${ }^{[12]}$.

At the Helmholtz-Institute and the Institute of Optics and Quantum Electronics in Jena, Germany, the POLARIS laser system $^{[13]}$ has been developed and commissioned during the past decade. It has commenced its daily operation. Within its experimental program, more than 16,000 shots have been delivered on target during the past two years. The POLARIS project was started in 1999 in order to develop a high-intensity HECDPSSL which could be used in laser-matter interaction experiments. The current key parameters of POLARIS are: $1030 \mathrm{~nm}$ centre wavelength, up to $6.5 \mathrm{~J}$ pulse energy ( $4 \mathrm{~J}$ on target), $144 \mathrm{fs}$ pulse duration, $7.1 \mu \mathrm{m}^{2}$ focal-spot size, and a temporal contrast for the 


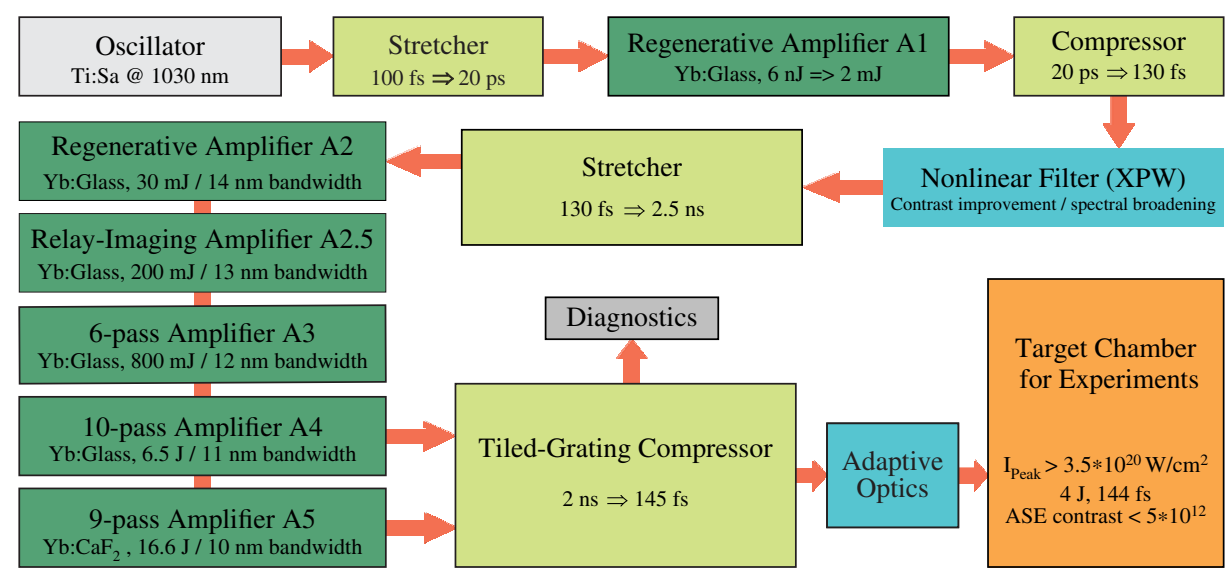

Figure 1. Schematic overview of the POLARIS laser system. An oscillator and two stretcher-compressor stages are used together with six amplifiers (green boxes). A nonlinear filter based on XPW broadens the spectrum and enhances the temporal contrast. An adaptive optics system is used to flatten the wavefront before the pulses enter the target chamber for focusing.

amplified spontaneous emission (ASE) of $5 \times 10^{12}$. With these parameters, a peak intensity of $3.5 \times 10^{20} \mathrm{~W} \mathrm{~cm}^{-2}$ is available for experiments. To the best of our knowledge POLARIS is currently the most powerful and intense diodepumped laser system. Nevertheless, the laser is still under continued development in order to further increase the pulse energy, decrease the pulse duration, and to better meet the requirements of experiments.

In this paper we describe the architecture of POLARIS, including the recently commissioned amplifier $\mathrm{A} 5^{[14]}$ and a newly installed stretcher-compressor system (double$\mathrm{CPA}^{[15]}$ ). After the first CPA stage, the pulses are used to generate a cross-polarized wave $\left(\mathrm{XPW}^{[16]}\right)$, thereby significantly improving the temporal intensity contrast. Furthermore, we present a detailed characterization of the amplified, compressed and focused pulses with respect to their temporal and spatial properties. The performance of the laser system is finally quantified for application in highintensity experiments in terms of peak intensity, temporal contrast and shot-to-shot stability.

\section{Architecture of the POLARIS laser}

In Figure 1 the layout of the POLARIS laser is shown. The system utilizes two subsequent CPA units and six amplification stages to amplify the laser pulses.

After pulse compression a radiation-shielded bunker with a target chamber is available for experiments. The seed pulses for the laser chain are generated in a commercial mode-locked Ti:sapphire oscillator (Coherent MIRA 900) running at a central wavelength of $1030 \mathrm{~nm}$ with a pulse energy of $7 \mathrm{~nJ}$ and a spectral bandwidth of $20 \mathrm{~nm}$ (FWHM). Before entering the first regenerative amplifier the pulses are temporally stretched to $20 \mathrm{ps}$. The amplifier A1 increases the pulse energy to $2 \mathrm{~mJ}$. Afterwards the pulses are re- compressed to $130 \mathrm{fs}$ before they enter the nonlinear XPWfilter realized by a $\mathrm{BaF}_{2}$-crystal.

The contrast-cleaned pulses are then stretched once more by the second stretcher (cf. $[13,17]$ ) to a pulse duration of $2.5 \mathrm{~ns}$ and furtheramplified by the second regenerative amplifier A2 to a pulse energy of $30 \mathrm{~mJ}$. The amplification to the Joule-level is accomplished with a relay-imaging amplifier (A2.5: $\left.E_{\text {out }}=200 \mathrm{~mJ}\right)$ and two multipass nonimaging amplifiers (A3: $E_{\text {out }}=800 \mathrm{~mJ}^{[18]}$, and A4: $E_{\text {out }}=$ $6.5 \mathrm{~J}$ ). In all of these amplifiers $\mathrm{Yb}^{3+}$-doped fluoridephosphate glass ${ }^{[19-22]}$ is used as the active material.

The amplified pulses can then either be sent to the main compressor or used as a seed for the final amplifier A5. This amplifier uses $\mathrm{Yb}: \mathrm{CaF}_{2}$ in a nine-pass configuration as the active material ${ }^{[23-25]}$ and is currently able to deliver a maximum output energy of $16.6 \mathrm{~J}$ (with $2.7 \mathrm{~J}$ seed energy). The active material of this amplifier is pumped by 120 laser diode stacks in a $2.5 \mathrm{~ms}$ long pump pulse at $940 \mathrm{~nm}$ with a $300 \mathrm{~kW}$ pump power. A detailed technical description of this amplifier is given in ${ }^{[14]}$. The beam line for the compression and focusing of the A5-amplified pulses is currently under development and will be finished soon.

For all the experiments shown here, only pulses amplified up to A4 were used. They were compressed with a tiledgrating compressor ${ }^{[26]}$ followed by an adaptive optics system to improve the focusability of the beam. By focusing the beam in the target chamber with a $f / 2$ parabola, a peak intensity in excess of $3.5 \times 10^{20} \mathrm{~W} \mathrm{~cm}^{-2}$ is available.

\section{Double-CPA and XPW for temporal contrast im- provement and spectral broadening}

Since the temporal intensity contrast has been shown to be one of the most important parameters for the laser's successful application in high-intensity experiments, we have 
continuously optimized the contrast of POLARIS. To apply a nonlinear filtering via XPW generation for contrast improvement, the front end was extended by the above-mentioned picosecond-CPA stage.

The two-pass Öffner-type stretcher consists of a $50 \times$ $50 \mathrm{~mm}^{2}$ gold grating with a line density of 1200 lines per $\mathrm{mm}$, a $50.8 \mathrm{~mm}$ concave mirror with a focal length of $200 \mathrm{~mm}$, and a convex mirror with a focal length of $-100 \mathrm{~mm}$. Back reflection from a hollow-roof mirror accomplishes the second pass. Due to the small stretching factor the footprint of the whole setup is only $40 \times 20 \mathrm{~cm}^{2}$.

In the subsequent regenerative amplifier A1 the stretched 20 ps pulses are amplified up to energies of $3 \mathrm{~mJ}$ without any spectral distortion due to self-phase modulation. The output spectrum of the amplifier has a bandwidth (FWHM) of $12 \mathrm{~nm}$, supporting a re-compressed pulse duration of $130 \mathrm{fs}$ if bandwidth-limited Gaussian pulses are assumed. To avoid the generation of post- and pre-pulses the round-trip time of the amplifier was matched to the pulse repetition time of the oscillator ${ }^{[27]}$. The beam profile of the output pulse has a smooth Gaussian shape (FWHM-diameter of $1 \mathrm{~mm}$ ) without any hotspots.

Because the nonlinear filter requires an unstreched bandwidth-limited input, the pulse has to be re-compressed after amplification in A1. The compression is achieved by a grating compressor, which consists of two gold gratings similar to the one in the stretcher. The gratings are separated by $95 \mathrm{~mm}$ and again a hollow-roof mirror is used for the second pass. The compressor can be operated in air. Pulses with a duration of $130 \mathrm{fs}$, which is the bandwidth limit of the amplified spectral intensity profile, are achieved.

After this compression the pulse is sent to the XPW-stage, where a $2 \mathrm{~mm}$ thick holographic cut $\mathrm{BaF}_{2}$-crystal is used as the nonlinear element. The beam is focused and recollimated with two lenses, both of them having a focal length of $1 \mathrm{~m}$. The $\mathrm{BaF}_{2}$-crystal can be rotated around its surface normal for the XPW optimization.

In order to adjust the required intensity the crystal is placed slightly behind the focal plane. Placing the whole focused beam between the two lenses, including the crystal and its mount, in a vacuum chamber avoids nonlinear effects in air. To separate the XPW and the input signal the setup is placed between two crossed polarizers with a extinction ratio better than $2 \times 10^{-6}$. Thus, considering a $6 \%$ conversion efficiency, an increase of the intensity contrast of more than four orders of magnitude is achievable.

The generated cross-polarized signal beam exhibits a $\mathrm{TEM}_{00}$-mode with a maximum pulse energy of $140 \mu \mathrm{J}$, and a spectral bandwidth of $21 \mathrm{~nm}$ (FWHM). It is used in the following as the seed for the remaining amplifier chain after passing the main ns-stretcher (Figure 1). Since due to the XPW process the spectrum of the seed pulses was broader than the bandwidth achieved with the conventional amplification-only setup (cf. [13]), a shorter pulse duration after the final compression could be achieved.
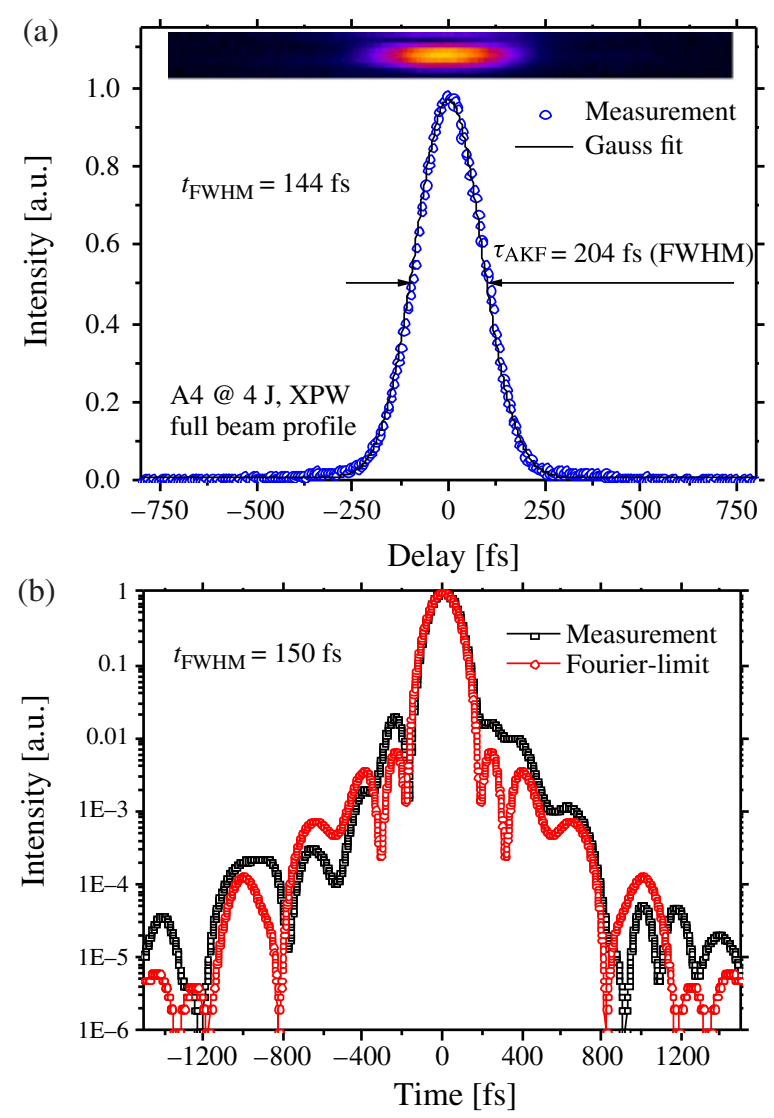

Figure 2. Pulse duration measurements of the compressed pulses. (a) Second-order autocorrelation of a $4 \mathrm{~J}$ pulse energy laser pulse which was amplified with A4. A Gaussian distribution fits well to the measured data. Blue: measurement, black: Gaussian fit. (b) High-dynamic Wizzler measurement of the $\mathrm{A} 2$ pulses (10 mJ pulse energy). Black: measurement, red: Fourier-limit.

\section{Pulse duration measurements}

In this section we present the temporal properties of the final compressed laser pulses.

In Figure 2(a) a measurement of the temporal profile of a laser pulse with $4 \mathrm{~J}$ energy is shown. This measurement was performed with a home-built second-order autocorrelator, where the pulse is focused in one dimension into a BBOcrystal using a cylindrical lens. The upper inset displays the spatially resolved autocorrelation signal. An FWHMpulse duration of $144 \mathrm{fs}$ is measured over the full beam profile. It fits well to a Gaussian pulse shape (black line). The broader spectrum of the XPW (cf. [13]) allows the pulse duration including amplification up to the amplifier A4 to be reduced from formerly 164 fs to currently $144 \mathrm{fs}$. In the former setup only a conventional, single-stage CPA layout was implemented.

In Figure 2(b) a high-dynamic measurement of the pulse duration of the compressed laser pulses (from the front end, i.e., up to amplifier A2) with $10 \mathrm{~mJ}$ pulse energy is shown. For this measurement a self-referenced spectral 
(a)

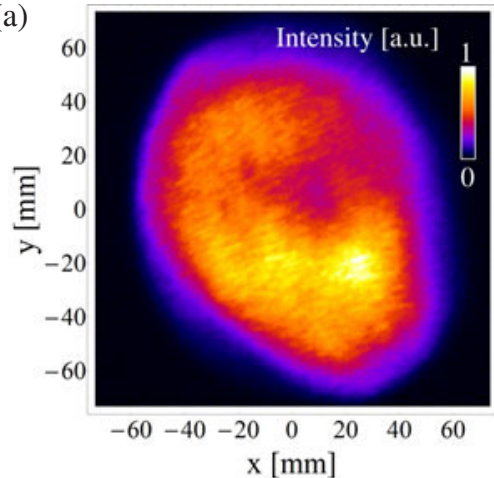

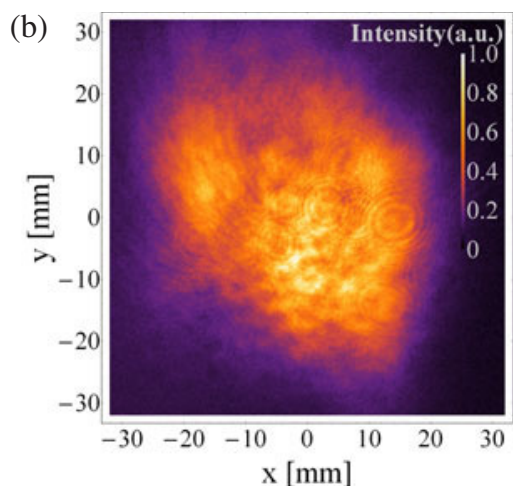

Figure 3. Measurements of the near-field intensity distribution of the amplified laser pulses. (a) Beam profile of the fourth amplifier (A4) measured in front of the focusing parabola after pulse compression. (b) Beam profile of the fifth amplifier (A5) measured directly after amplification with a pulse energy of $16.6 \mathrm{~J}$.

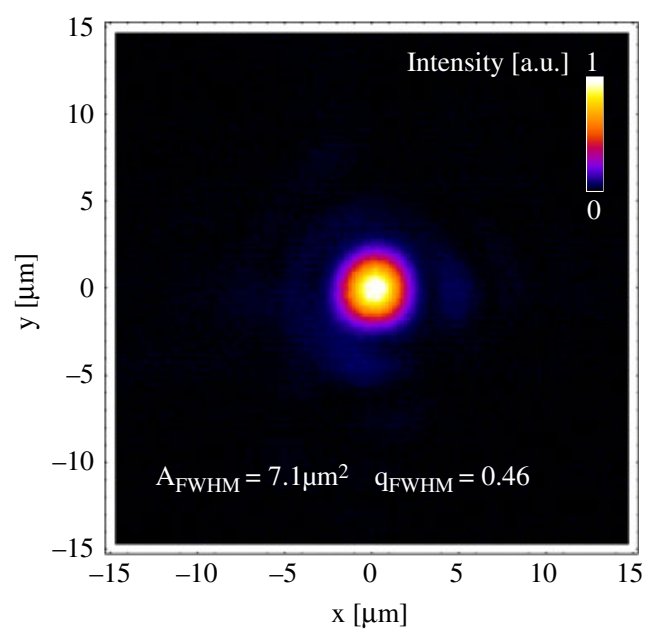

Figure 4. Measured transverse far-field profile of the A4-amplified and compressed laser pulses. The area within which the intensity is larger than $I_{\max } / 2$ is $7.1 \mu \mathrm{m}^{2}$ and contains $46 \%$ of the pulse energy $(q=0.46)$.

interferometer (Wizzler) was used and the spectral phase was flattened using a Dazzler (both Fastlite Techn.) positioned directly behind the oscillator without using the XPW-stage. This measurement highlights the capability of generating nearly transform-limited laser pulses with high temporal contrast. However, in order to use the Dazzler's spectral shaping capability together with the XPW-cleaned pulses the device needs to be inserted behind the XPW-stage.

\section{Spatial pulse characterization}

A homogeneous super-Gaussian-like near-field profile of the laser pulses is desirable for an efficient energy extraction and a maximum compressor throughput. The maximum extractable pulse energy of the main amplifiers A4 and A5 is limited by the fluence threshold at which laser-induced damage occurs on the surface or in the volume of the active material. The Yb:glass, which is used in amplifier A4, withstands a fluence of $3 \mathrm{~J} \mathrm{~cm}^{-2}$ in long-term operation, whereas the fluence on the $\mathrm{Yb}: \mathrm{CaF}_{2}$-crystal used in amplifier A5 is currently limited by the damage threshold of the AR coatings to $2 \mathrm{~J} \mathrm{~cm}^{-2}$. In Figure 3 the near-field intensity distributions are shown for pulses that are amplified in A4 and A5, respectively.

The measurement for the A4-amplified pulse in Figure 3(a) was done in front of the focusing parabola. Between the exit of the last amplifier and the compressor entrance the laser beam diameter is expanded by a $5.6 \times$ magnification telescope. The enlarged beam diameter ensures a safe operation in terms of laser-induced damage of the compressor gratings $\left(\mathrm{F}_{\text {damage }}=200 \mathrm{~mJ} \mathrm{~cm}^{-2}\right)$.

The beam profile of the A5-amplified pulses, shown in Figure 3(b), was recorded after the last passage through the active material. In order to realize a sufficient beam diameter for the pulse compressor a $2.5 \times$ magnification telescope is placed between the amplifier A5 and the pulse compressor.

To generate a high peak intensity for laser-matter experiments the pulses are focused in the target chamber with an off-axis parabola ( $f=300 \mathrm{~mm}, f / 2$, cf. Figure 1). Since pulses from the amplifier A5 have not been compressed yet, we have only been using the A4-amplified pulses for experiments so far. The compression and focusing of the A5 pulses is currently under investigation.

For the improvement of the focusability of the pulses from A4 we installed an adaptive optics system. The combination of a 160-mm-diameter adaptive mirror and a wavefront sensor helps to flatten the wavefront and to reduce the focalspot size as well as to increase the energy content within the focal-spot area (for details see [13]).

The focal spot of the amplified laser pulses is shown in Figure 4. For this measurement the amplified pulses were strongly attenuated in the laser chain. The area within which the intensity is larger than $I_{\max } / 2\left(A_{\mathrm{FWHM}}\right)$ is $7.1 \mu \mathrm{m}^{2}$ and contains $46 \%$ of the total laser pulse energy $\left(q_{\mathrm{FWHM}}=0.46\right)$. The Strehl ratio, defined as the ratio of the achieved peak intensity to the calculated peak intensity assuming a flat wavefront, is 0.45 and likely limited by residual chromatic and wavefront aberrations. 


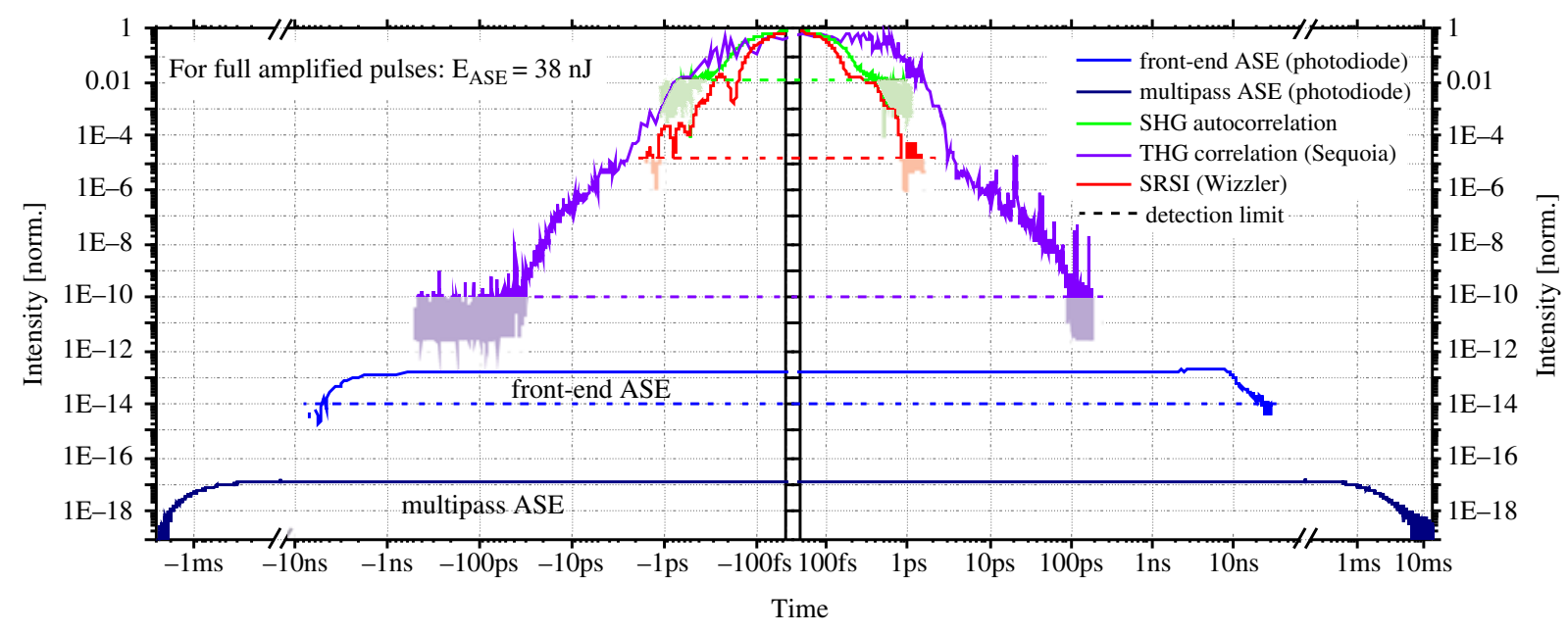

Figure 5. High-dynamic temporal characterization of the amplified and compressed laser pulses. The intensity is given as the relative on-target intensity using $f / 3$-focusing and negative times are defined as the times before the laser pulse. The laser pulse is characterized using different measurements: red: Self-Referenced Spectral Interferometry (Wizzler), green: SHG-correlator, pink: THG-correlator, blue and dark blue: photodiode. The detection limit of each measurement is marked as a dashed line.

\section{High-dynamic temporal pulse characterization}

As mentioned in the introduction, the temporal intensity contrast is one of the most important parameters for the application of high-intensity laser pulses in experiments. In this section we will quantify the temporal contrast by giving an overview of the temporal behaviour of the compressed pulses on different timescales with a high-dynamic range.

In Figure 5 the temporal characterization of the POLARIS pulses is shown on a log-log scale. The graph combines different types of measurements for the main laser pulse, the front-end ASE and the multipass-amplifier ASE. The relative on-target intensity has been recorded using the above-mentioned $f / 2$-focusing parabola currently installed in our target chamber. Due to the different methods used, the characterization spans both a range of 17 orders of magnitude for the relative intensity and a time-window from femtoseconds to milliseconds. The individual detection limits for each individual measurement are indicated by dashed lines in the corresponding colours.

The red (Wizzler) and green (SHG-autocorrelation) curves in Figure 5 are the pulse duration measurements which are shown above. They are able to measure the temporal intensity profile of the laser pulse with a dynamic range of 1.5 (SHG-autocorrelator) and 5 (Wizzler) orders of magnitude.

To further resolve the temporal structure of the compressed laser pulses a commercial third-order cross-correlator (Sequoia, Amplitude Technologies) has been used. It is able to resolve 10 orders of magnitude relative intensity for pulses centred at $1030 \mathrm{~nm}$. Here we mention that the measurement with the Sequoia is taken in the near-field of the compressed pulse and since we are using a tiled-grating compressor some spectral components of the pulses are missing depending on the lateral position in the laser pulse ${ }^{[17]}$. This may lead to a smaller relative intensity contrast for the laser pulse (and its leading edge) as compared to the contrast in the focal spot where the pulses are applied in experiments.

However, even the dynamic range of the third-order crosscorrelator is not sufficient to resolve the relative intensity contrast of our pulses when using the XPW front end. Apart from some residual pre-pulses, the relative contrast ratio of the pulses can no longer be resolved for $t<-40$ ps before the main pulse due to the detection limit of $10^{-10}$ for the relative intensity contrast. The residual pre-pulses are likely generated by reflections inside the amplifier chain ${ }^{[28]}$. Their elimination is the subject of ongoing work.

The ASE of the amplifiers was measured by operating the laser with fully pumped amplifiers, but blocking pulses from the oscillator. Due to the non-saturated amplification within all amplifiers of POLARIS the generated ASE without seed is identical to the ASE generated during the amplification. Using a high-sensitivity photodiode and calibrated ND filters we are able to measure the temporally dependent relative intensity of the ASE. Furthermore, with our focal-spot diagnostic we could record the spatial far-field distribution of the ASE contributions from the different amplifiers, finally leading to the relative ASE intensities on target. This method is described in detail in ${ }^{[29]}$.

Due to its architecture the POLARIS laser emits two different types of ASE. The first is the so-called front-end ASE which is generated in the first amplifier (A1), reduced by the XPW-stage but further amplified by the second amplifier, with a pulse duration of $13 \mathrm{~ns}$, which corresponds to the round-trip times in the regenerative amplifiers. The blue curve in Figure 5 displays a photodiode measurement of the front-end ASE having a intensity of $2 \times 10^{-13}$ relative to the main pulse. 
The second part is the so-called multipass-ASE, which is generated in the multipass amplifiers A2.5, A3, and A4. Due to their multipass architecture, which does not implement a cavity, the pulse duration is on the order of a few milliseconds. This corresponds to the pump duration and the fluorescence lifetime of the active material.

Note that the front-end ASE is also amplified in the multipass amplifiers since this front-end ASE is seeding - together with the main laser pulse - all subsequent amplifiers. Due to the long pulse duration of $2 \mathrm{~ms}$, the multipass-ASE energy and intensity can be significantly decreased by temporal gating of the main laser pulse during the amplification. This is accomplished by two Pockels cells, which are installed before and after the amplifier A4. Their gate duration is set to be as short as $10 \mathrm{~ns}$.

The relative intensity of the multipass-ASE with respect to the main pulse is $1.5 \times 10^{-17}$ and displayed in Figure 5 as the dark blue line. This measurement was taken with a photodiode where the amplifier chain was blocked in front of amplifier A2.5. Due to the missing cavities in the multipass amplifiers the focusability of the multipass-ASE is strongly degraded and leads to a one order of magnitude larger focal spot as compared to the main pulse. Since the front-end ASE is generated by two regenerative amplifiers, which define the spatial profile and divergence of the main pulse, they both have a nearly identical focal-spot size.

Additionally, we measured the energy of the ASE to be $38 \mathrm{~nJ}$, which is the sum of all contributions. Compared to an ASE energy of $130 \mu \mathrm{J}$ and a relative ASE intensity of $10^{-9}$ of our conventional and formerly used front end (without XPW, cf. [13]) we have significantly improved the experimental performance in terms of temporal intensity contrast with the double-CPA and XPW front end. As a consequence, up to $2 \mathrm{~ns}$ before the main laser pulse a contrast of $5 \times 10^{12}$ (relative intensity of $2 \times 10^{-13}$ ), and up to $1 \mathrm{~ms}$ a temporal intensity contrast of $7 \times 10^{16}$ (relative intensity of $1.5 \times 10^{-17}$ ) has been achieved.

\section{Experimental performance}

With the currently installed $f / 2$-focusing parabola the POLARIS laser is capable of delivering a peak intensity of $3.5 \times 10^{20} \mathrm{~W} \mathrm{~cm}^{-2}$ with a repetition rate of $1 / 40 \mathrm{~Hz}$. The pulses are as short as $144 \mathrm{fs}$, with a pulse energy of $4 \mathrm{~J}$ on target.

With the final commissioning of amplifier A5, the ontarget pulse energy will likely exceed $10 \mathrm{~J}^{[14]}$. To reproducibly operate the laser on a daily basis we have optimized and installed enclosures to minimize air fluctuations and reduce dust contamination on the optics. Furthermore, most of the optics which were used for online alignment (e.g., thermal drift compensation) are motorized for remote control. In Figure 6(a), a measurement of the pulse duration of 300 consecutive (taken over $3.3 \mathrm{~h}$ during an experiment)
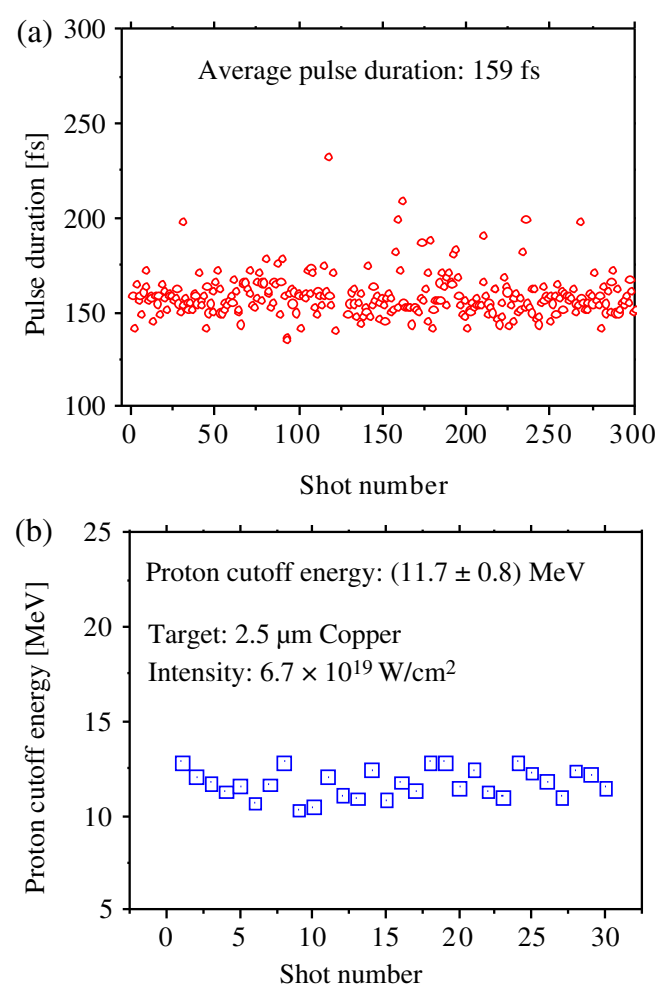

Figure 6. Stability measurements. (a) Pulse duration measurement with a single-shot SHG-autocorrelator during 300 consecutive full energy shots (taken over $3.3 \mathrm{~h}$ ). (b) Cutoff energy of TNSA-accelerated protons from a 2.5 - $\mu \mathrm{m}$-thick copper foil versus shot number (30 consecutive shots).

full energy shots is shown. The sometimes increased pulse duration is likely induced by mechanical vibrations on the very sensitive tiled-grating arrangement in the pulse compressor.

Up to now we have performed several experimental campaigns over the past two years in order to accelerate protons or electrons. As an example, protons have been accelerated from thin foils by the so-called target-normal sheath acceleration $\left(\mathrm{TNSA}^{[4]}\right)$. To investigate the stability of the entire laser system we have used such an experiment to record 30 consecutive shots over a time of $20 \mathrm{~min}$ while measuring the cutoff energy of the accelerated protons. In Figure 6(b) the data is displayed. In this particular experiment a $2.5-\mu \mathrm{m}$-thick copper foil was used as target and an average proton energy of $11.7 \mathrm{MeV}$ was achieved. While keeping all parameters constant during these 30 shots the standard deviation for the measured proton energy was as small as $7 \%$. Note that for our parameters the proton cutoff energy only weakly depends on the pulse duration ${ }^{[30]}$.

\section{Conclusion and outlook}

In conclusion we present an overview of the fully diodepumped solid-state laser POLARIS. With a peak intensity of $3.5 \times 10^{20} \mathrm{~W} \mathrm{~cm}^{-2}$ it is currently, to the best of our knowledge, the most intense DPSSL worldwide. Moreover, 
the pulses are generated with an ultra-high temporal contrast of $5 \times 10^{12}$ for the ASE of the laser system.

Furthermore, we have shown that the pulse energy can be increased with a diode-pumped $\mathrm{Yb}: \mathrm{CaF}_{2}$-crystal to $16.6 \mathrm{~J}$. In the near future, the pulses from this amplification stage will be compressed and focused to be available for high-intensity laser-matter experiments.

Finally, we investigate the operation performance of POLARIS in laser-matter interaction experiments, where a stable generation of protons has been achieved with a standard deviation of $7 \%$ for the cutoff energy.

\section{Acknowledgements}

The research leading to these results has received funding from the European Commission's (EC) 7th Framework Programme (LASERLAB-EUROPE, grant no. 228334) and from the Bundesministerium für Bildung und Forschung (BMBF) (03ZIK445 and 03Z1H531).

\section{References}

1. D. Strickland and G. Mourou, Opt. Comm. 56, 219 (1985).

2. E. Esarey, C. B. Schroeder, and W. P. Leemans, Rev. Mod. Phys. 81, 1229 (2009).

3. H. T. Kim, K. H. Pae, H. J. Cha, I. J. Kim, T. J. Yu, J. H. Sung, S. K. Lee, T. M. Jeong, and J. Lee, Phys. Rev. Lett. 111, 165002 (2013).

4. A. Macchi, M. Borghesi, and M. Passoni, Rev. Mod. Phys. 85, 751 (2013).

5. S. Corde, K. T. Phuoc, A. Beck, G. Lambert, R. Fitour, E. Lefebvre, V. Malka, and A. Rousse, Rev. Mod. Phys. 85, 1 (2013).

6. Y. Izawa, N. Miyanaga, J. Kawanaka, and K. Yamakawa, J. Opt. Soc. Korea 12, 178 (2008).

7. H. Johnston, Physicsworld 26, 50 (2013).

8. M. Siebold, J. Hein, M. Hornung, S. Podleska, M. C. Kaluza, S. Bock, and R. Sauerbrey, Appl. Phys. B 90, 431 (2008).

9. T. Goncalves-Novo, D. Albach, B. Vincent, M. Arzakantsyan, and J. C. Chanteloup, Opt. Exp. 21, 855 (2013).

10. S. Banerjee, K. Ertel, P. D. Mason, P. J. Phillips, M. Siebold, M. Löser, C. Hernandez-Gomez, and J. L. Collier, Opt. Lett. 37, 2175 (2012).

11. A. Bayramian, P. Armstrong, E. Ault, R. Beach, C. Bibeau, J. Caird, R. Campbell, B. Chai, J. Dawson, C. Ebbers, A. Erlandson, Y. Fei, B. Freitas, R. Kent, Z. Liao,
T. Ladran, J. Menapace, B. Molander, S. Payne, N. Peterson, M. Randles, K. Schaffers, S. Sutton, J. Tassano, S. Telford, and E. Utterback, J. Fusion Sci. Technol. 52, 383 (2007).

12. T. Töpfer, J. Neukum, J. Hein, and M. Siebold, Laser Focus World 46, 64 (2010).

13. M. Hornung, S. Keppler, R. Bödefeld, A. Kessler, H. Liebetrau, J. Körner, M. Hellwing, F. Schorcht, O. Jäckel, A. Sävert, J. Polz, A. K. Arunachalam, J. Hein, and M. C. Kaluza, Opt. Lett. 38, 718 (2013).

14. A. Kessler, M. Hornung, S. Keppler, F. Schorcht, M. Hellwing, H. Liebetrau, J. Körner, A. Sävert, M. Siebold, M. Schnepp, J. Hein, and M. C. Kaluza, Opt. Lett. 39, 1333 (2014).

15. M. P. Kalashnikov, E. Risse, H. Schönnagel, A. Husakou, J. Herrmann, and W. Sandner, Opt. Exp. 12, 5088 (2004).

16. A. Jullien, O. Albert, G. Chériaux, J. Etchepare, S. Kourtev, N. Minkovski, and S. M. Saltiel, J. Opt. Soc. Am. B 22, 2635 (2005).

17. M. Hornung, R. Bödefeld, M. Siebold, A. Kessler, M. Schnepp, R. Wachs, A. Sävert, S. Podleska, S. Keppler, J. Hein, and M. C. Kaluza, Appl. Phys. B 101, 93 (2010).

18. J. Hein, S. Podleska, M. Siebold, M. Hellwing, R. Bödefeld, R. Sauerbrey, D. Ehrt, and W. Wintzer, Appl. Phys. B 79, 419 (2004).

19. T. Töpfer, J. Hein, J. Philipps, D. Ehrt, and R. Sauerbrey, Appl. Phys. B 71, 203 (2000).

20. T. Töpfer, J. Hein, W. Wintzer, D. Ehrt, and R. Sauerbrey, Glass Sci. Technol. 75, 223 (2002).

21. D. Ehrt, Curr. Opin. Solid State Mater. Sci. 7, 135 (2003).

22. S. Paoloni, J. Hein, T. Töpfer, H. G. Walther, R. Sauerbrey, D. Ehrt, and W. Wintzer, Appl. Phys. B 78, 415 (2004).

23. M. Siebold, M. Hornung, R. Bödefeld, S. Podleska, S. Klingebiel, C. Wandt, F. Krausz, S. Karsch, R. Uecker, A. Jochmann, J. Hein, and M. C. Kaluza, Opt. Lett. 33, 2770 (2008).

24. M. Siebold, S. Bock, U. Schramm, B. Xu, J. L. Doualan, P. Camy, and R. Moncorge, Appl. Phys. B 97, 327 (2009).

25. J. Körner, C. Vorholt, H. Liebetrau, M. Kahle, D. Klöpfel, R. Seifert, J. Hein, and M. C. Kaluza, J. Opt. Soc. Am. B 29, 2493 (2012).

26. M. Hornung, R. Bödefeld, M. Siebold, M. Schnepp, J. Hein, R. Sauerbrey, and M. C. Kaluza, Appl. Opt. 46, 7432 (2007).

27. S. Keppler, R. Bödefeld, M. Hornung, A. Sävert, J. Hein, and M. C. Kaluza, Appl. Phys. B 104, 11 (2011).

28. S. Keppler, M. Hornung, R. Bödefeld, M. Kahle, J. Hein, and M. C. Kaluza, Opt. Exp. 20, 20742 (2012).

29. S. Keppler, M. Hornung, R. Bödefeld, A. Sävert, H. Liebetrau, J. Hein, and M. C. Kaluza, Opt. Express 22 (9), (2014) (accepted for publication).

30. J. Schreiber, F. Bell, F. Grüner, U. Schramm, M. Geissler, M. Schnürer, S. Ter-Avetisyan, B. M. Hegelich, J. Cobble, E. Brahmbrink, J. Fuchs, P. Audebert, and D. Habs, Phys. Rev. Lett. 97, 045005 (2006). 